\title{
Nursing in the Orthogeriatric Setting
}

\author{
Julie Santy-Tomlinson, Karen Hertz, \\ Charlotte Myhre-Jensen, and Louise Brent
}

\subsection{Introduction}

The aims of this chapter are to provide an overview of the role of nurses in the multidisciplinary orthogeriatric team and help medical, surgical practitioners and therapists to understand the potential of the nursing team to foster collaboration in achieving best outcomes for patients. The call to action issued by the Fragility Fracture Network [1] describes four pillars of fragility fracture care, all of which involve expert nursing: (1) multidisciplinary co-management in the acute fracture episode; (2) post-acute rehabilitation; (3) secondary prevention after every fragility fracture and (4) the formation of multidisciplinary national alliances. These pillars support the need for nursing education, collaboration between nurses and their supporting local, national and global associations and the development of education,

This chapter is a component of Part 5: Cross-cutting issues.

For an explanation of the grouping of chapters in this book, please see Chapter 1: "The multidisciplinary approach to fragility fractures around the world-an overview".

\author{
J. Santy-Tomlinson $(\bowtie)$ \\ Orthopaedic Nursing, Odense University Hospitals and University of Southern Denmark, \\ Odense, Denmark \\ e-mail: juliesanty@tomlinson15.karoo.co.uk \\ K. Hertz \\ University Hospitals of North Midlands, Stoke-on-Trent, UK \\ e-mail: Karen.Hertz@uhnm.nhs.uk \\ C. Myhre-Jensen \\ Department of Orthopaedic Surgery and Traumatology, Odense University Hospital, \\ Odense, Denmark \\ e-mail: Charlotte.Myhre.Jensen@rsyd.dk \\ L. Brent \\ National Office of Clinical Audit, Dublin, Ireland \\ e-mail: louisebrent@noca.ie


clinical guidance and resources that can be adapted and adopted in any part of the world so that best practice nursing care can be achieved. In some countries, regions and/or localities, the concept of orthogeriatric nursing has yet to be fully recognised. Even so, the principles of orthogeriatric care and management can be applied in any setting with consideration of the context of local practice, culture and resources.

\subsection{Nursing Care Quality}

Orthogeriatric patients have complex health care needs, many of which can be met by skilled, compassionate nursing. Nursing involves autonomous and collaborative care that includes the promotion of health as well as prevention of illness and supporting recovery during ill health and following injury and surgery. Nursing also embodies advocacy, promotion of safety, leadership and participation in shaping health policy [2]. Nurses make a unique contribution to orthogeriatric care because they spend the most time with patients and develop a therapeutic relationship with individuals and their families while acting as coordinators of care. They understand the patient experience of the fracture and its care through qualitative exploration of experiences. These experiences are often described as difficult and painful, leading to significant decrease in quality of life, and are fraught with restrictions and insecurity $[3,4]$, although patient experiences in resource-poor countries have yet to be studied or documented.

Hip fracture audit, where it takes place, has had a significant impact on the quality of medical and surgical care, but with a limited focus on nursing. It is essential that indicators of the value of nursing care are developed and measured [5]. The overall contribution of health care delivery is often measured in terms of health status, outcomes, readmissions rates, length of stay, complication rates and mortality [6], but these do not always help to capture the specific contribution of nursing care.

Nurse-sensitive quality indicators include patient comfort and quality of life, complication rates, safety, empowerment and satisfaction. More specific clinical indicators can include healthcare-associated infection, pressure ulcers, falls and drug administration errors [7]. Pain management, delirium, pressure ulcers, hydration and nutrition, constipation, prevention of secondary infections and venous thromboembolism (VTE), rehabilitation and remobilisation are all nursing care priorities [5] but evidence-based nurse management strategies need to coexist with medical and therapist models of care. This includes reducing the risk of complications and mortality, improving recovery, maintaining function and improving patient experiences. Preventing harm and patient safety, focused on avoiding adverse events, are multidisciplinary concerns that vary depending on the country and its resources. Nurses, through their extended contact with patients, remain central to recognising risk and alerting other team members. Nursing quality indicators should also take into consideration the local context and available resources. 


\subsection{Acute Care}

The high prevalence, complexity of needs, length of stay and cost, often make hip fracture care the focus of practice development. The skills and knowledge needed to look after patients with hip fractures, however, also apply to the management of all older people with fractures and include fundamental aspects of nursing care for the adult as well as specialised interventions for older people [8, 9]. A hip fracture is a sudden traumatic event, threatening many aspects of patients' lives and a forceful reminder of their mortality [10]. Care is dominated by restoring function, so physical care attracts the most attention and the primary goal of nursing is to maximise mobility and preserve optimal function whilst also prioritising psychosocial factors so that patients can participate in their rehabilitation [11].

Emergency care following hip fracture usually takes place in Emergency Departments that are noisy, busy and overstimulating, making them inappropriate care environments for vulnerable older people in a state of personal and physical crisis. Avoiding the impact of this situation requires consideration of three principles [12]:

- Timeliness - avoiding unnecessary and unwanted delay.

- Effectiveness - aiming for optimal outcomes using the best available evidence.

- Patient-centeredness - care that is respectful of and responsive to individual needs.

This is equally true of the hospital ward and perioperative environment which are rarely designed to meet the needs of frail older people.

\subsubsection{Complexity and Frailty}

Trauma care for older people must follow the same principles as for all age groups but the normal and abnormal changes of ageing, compounded by active comorbidities, mean that morbidity and mortality are increased concerns [13].

Trauma/orthopaedic services evolved to treat all adult patients, irrespective of age, following all types of musculoskeletal injury. However, there has been less focus on the complex needs of those who are older and frail but have also sustained a fracture. Highly skilled nursing is needed that is tailored to the needs of the older person. This must encompass expert care of both the older person and the individual with a musculoskeletal condition/injury.

These "complex patients" present with multiple problems including; comorbidity, multimorbidity, poly-pathology, dual diagnosis and multiple chronic conditions [14] with multiple interlocking problems related to both breadth (range) and depth (severity) [15]. In the orthogeriatric patient there are three main facets of complexity; the person, the fracture and the care environment - all of which have a significant impact on patient care outcomes and are influenced by nursing care (see Fig. 17.1). 


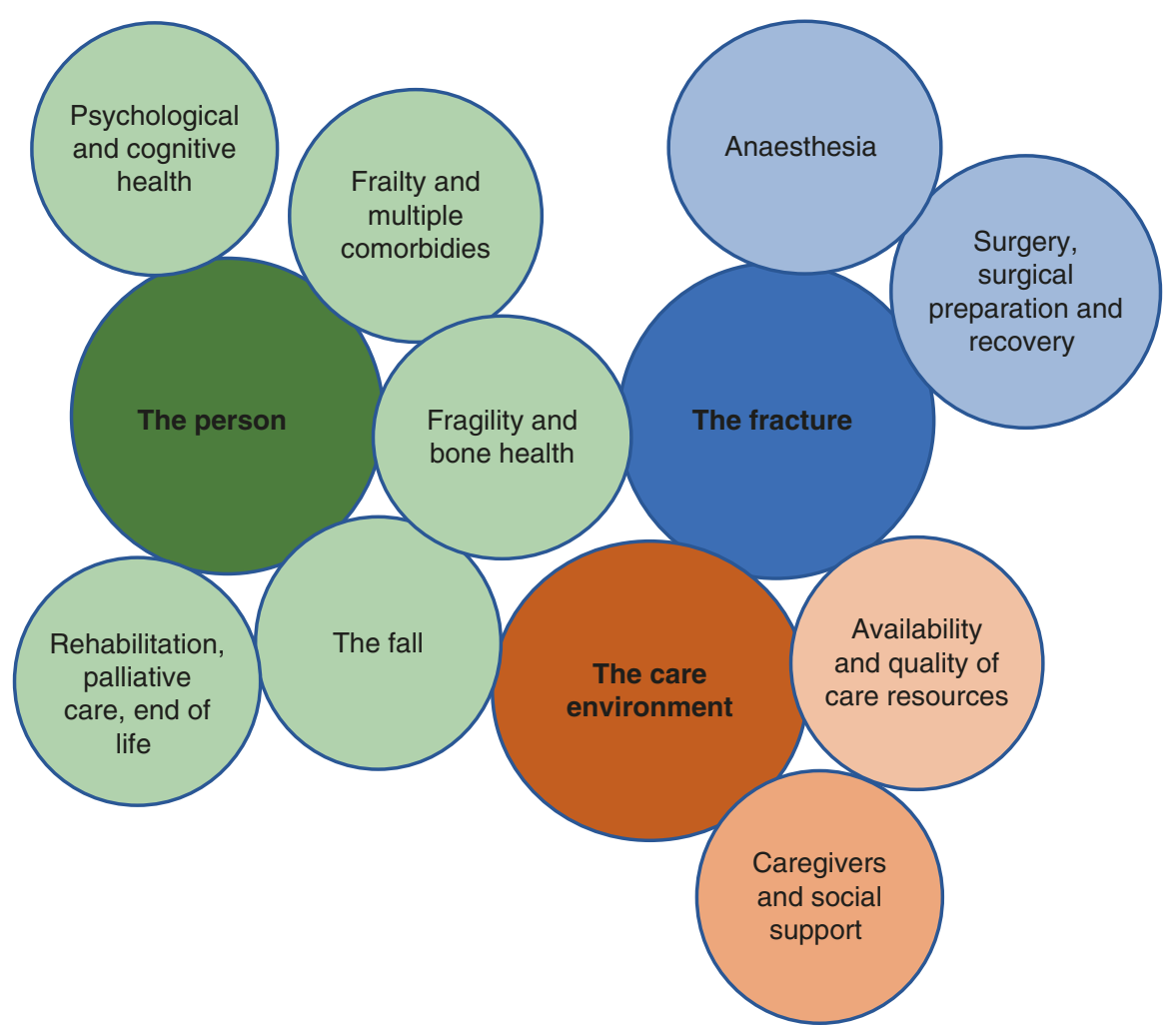

Fig. 17.1 The complexity of nursing care needs for hospitalised patients with hip fracture (adapted from Hertz \& Santy-Tomlinson 2017 [16])

Complexity and frailty are linked. As described in Chap. 4, frailty is a distinctive health state in which multiple body systems gradually lose their in-built reserves; incidence increases with age and is associated with negative outcomes such as falls, hospitalisation, poor functional outcomes and death [17]. Dramatic changes in their physical and mental well-being after any event which challenges a frail person's health, such as an injury or surgery leads to increased vulnerability and diminished resistance to stressors [18]. Frailty can be physical or psychological or a combination of the two elements when physical frailty is coupled with cognitive impairment [19]. Frailty is known to be a dynamic state, varying in severity with capacity to be made better and worse. It is not an inevitable part of ageing; but is a long-term condition that is linked with falls and fractures.

The identification of frailty is an interdisciplinary responsibility, but nurses need to recognise it in patient assessment so that care can be planned accordingly [20]. In collaboration with the orthogeriatric team, a validated assessment tool may identify frailty and its contributing factors as part of the multidisciplinary assessment process (see Chap. 4). 
Sarcopenia, reduced muscle mass and strength, is an additional driver for specialised nursing care for orthogeriatric patients, increasing fracture risk as it contributes to falls and is associated with lower bone mineral density, partly due to reduced forces of muscle on bone [17]. This combination of problems affects balance, gait and overall ability to perform tasks of daily living, highlighting the link between frailty, falls and fragility. Just as osteoporosis predicts the future risk of fracture, sarcopenia is a powerful predictor of future disability and need for specialised nursing care to reverse or manage decline.

\subsubsection{Nursing Assessment and Management of Pain}

Frequent, accurate pain assessment is essential. Pain in older people is often underreported by patients and ignored by health care professionals. Unmanaged or undermanaged pain increases the risk of delirium, impaired mobility, chronic pain and poorer long-term function [21]. Cognitive impairment increases the risk of pain not being recognised. The individual and highly variable nature of pain and an individual's response to it make accurate assessment central to nursing care that facilitates individualised pain management and monitoring. If pain is poorly controlled, mobilisation will be delayed, increasing the risk of complications of prolonged immobility, increased dependency and delirium.

Verbal reports of pain are valid and reliable in patients with mild to moderate dementia or delirium but the assessment of pain in a patient with more severe cognitive impairment may be more difficult. It has been shown that cognitively impaired and acutely confused patients receive less analgesia than their unimpaired counterparts. Using specific assessment tools can help staff to understand the individual needs of a person with dementia and encourages families to share patient information, characteristics and behaviour that enable staff to better understand their pain experience and needs [22]. For pain assessment to be effective it must be carried out frequently and recorded accurately, just like vital signs or the administration of medication and other interventions. Pain management must provide sufficient relief to allow nursing care to be performed with least distress to the patient followed by reassessment and appropriate administration of analgesia.

As nurses become increasingly responsible for more advanced patient care interventions, non-medical prescribing will permit nurses to assess pain and to formulate a patient-centred plan for pain management. In some settings, advanced practitioners with enhanced skills can prescribe a range of medications including opioid and non-opioid analgesics to enable a faster response to patient needs. Administration pre-operatively of nerve blocks for patients with hip fracture is becoming increasingly common, minimising the need for opiates which have multiple risk factors in older and frail patients and have been shown to have a significant positive effect on the pain experience [23]. 


\subsubsection{Nursing Assessment and Management of Delirium}

Delirium is a common and serious condition in older surgical patients, with an incidence of up to $60 \%$ following hip fracture. It is identifiable by its sudden onset, fluctuating course and effect on consciousness and perception. The consequences of delirium can include [24]:

- Increased rates of complications.

- Increased length of hospital stay.

- Increased risk of dementia.

- More care and support needs following discharge.

- Increased risk of death.

Assessment of delirium has two focuses:

1. Identification of those at highest risk.

2. Identification of those developing symptoms of delirium.

Delirium is avoidable and the severity can be reduced through nursing interventions as the nursing team are most likely to recognise the signs of delirium discussed in Chap. 11. Good communication with patients, family and carers can help practitioners to recognise risk of delirium and subtle changes that suggest both delirium and its underlying causes, enabling them to co-ordinate multidisciplinary interventions.

Patients with or at risk of delirium and their carers need information about delirium and what they might experience, encouraging them to report changes and inconsistencies in behaviour as there are several nursing interventions which may prevent delirium [25] as well as contribute to effective general nursing care of all older people:

- An environment that helps to re-orientate patients; large-face clocks and calendars, well-lit areas with clear signage.

- Gentle re-orientation by introducing team members and with explanations of time and place; family/friends should be encouraged to visit and be supported in modifying their own communication.

- Dehydration, hypoxia and constipation prevention and management.

- Supported mobilisation to give more control.

- Recognition and management of infections.

- Assessment and management of pain.

- Ensure dentures are fitting correctly and encourage patients to eat.

- Resolve reversible causes of sensory impairment e.g. hearing and visual aids.

- Facilitating sleep and rest. 


\subsubsection{Pressure Ulcer Prevention}

Pressure ulcers (PUs) are serious complications of immobility, hospitalisation and surgery which increase the need for nursing care and extend hospital stay. Patients with hip fracture are at high risk, so prevention and management are central to patient safety. PU prevention is largely a nursing responsibility, but a multidisciplinary approach is needed to manage all risk factors.

Assessment of the skin on admission should be followed by frequent reassessment. PUs can develop rapidly, so prompt and repeated assessment of risk using an appropriate and validated tool is essential in identifying the intrinsic and extrinsic factors that may lead to PUs. Identification of specific risk factors can then assist in planning and delivering appropriate interventions for prevention [26]. Examples of intrinsic and extrinsic factors are listed in Table 17.1.

Prevention strategies must be individualised to the patient's skin condition and risk factors and based on evidence-based guidelines [27]. Interventions should include:

- Head to toe skin assessment at least once during each nursing shift.

- Pressure relieving/redistributing support surfaces on beds and chairs, including in the home.

- Off-loading of bony prominences, especially the sacrum and heels.

- Frequent re-positioning based on assessment of the individual's tissue tolerance to pressure, including use of the 30-degree tilt to ensure off-loading of bony prominences.

- General skin care; careful washing and drying of the skin (especially following incontinence) and the use of emollient therapy to help promote the skin barrier function.

- Effective management of pain to promote movement and mobilisation.

- Nutrition and hydration.

Table 17.1 Common pressure ulcer risk factors for patients following hip fracture and surgery

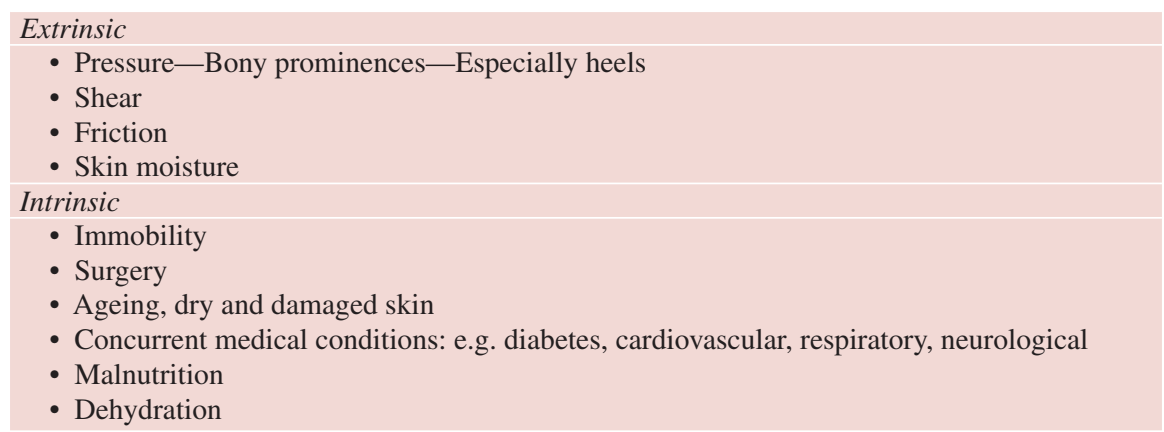


- Carefully selected support surfaces on beds and chairs;

- Foam mattresses to redistribute pressure and reduce friction in patients at medium risk of PUs.

- For patients at high to very high risk, dynamic pressure reducing equipment such as alternating pressure mattresses for all patients until their mobility has improved enough for them to be able to change their own position.

- Once remobilisation is in progress, these principles should also be applied to seating.

\subsubsection{Nutrition, Hydration, Acute Kidney Injury and Constipation}

Nutrition and hydration are fundamental to recovery and are the responsibility of the whole team, but the nursing team is central to adequate dietary and fluid intake because of their extended presence. Routine care must include assessment of nutritional status on admission, nutritional intake following admission, nurse-based strategies to improve calorific intake and, where appropriate, referral for dietetic advice (see Chap. 18). Team communication should maximise nutrition and fluid intake in close collaboration with patients/families. Minimal duration of preoperative fasting is a priority [8] so nurses need to be able to assess the likely time of commencement of surgery.

Fluid management in older people can be difficult; they may self-regulate fluid to try to control urinary incontinence/frequency and difficulties in accessing toilet facilities. Close monitoring of fluid balance is an essential aspect of nursing care to prevent or identify acute kidney injury. Patients' acceptance of drinks is often poor and nursing interventions to promote adequate fluid intake should include:

- Accurate administration of prescribed IV and parenteral fluids.

- Avoidance of long periods of fasting, including interdisciplinary commitment to ensuring the timing of older patients' surgery is managed and transparent.

- Assisting with oral fluid intake with regard for patient preferences and monitoring fluid intake/output.

- Appropriate toilet signage, regular toileting and other measures to enable patients to maintain continence.

- Close observation of vital signs and other indicators of health deterioration.

Renal function is affected by the ageing process as well as ill health. Acute kidney injury (AKI) is a significant cause of death in hospitalised older people: a sudden episode of kidney failure or damage that happens rapidly and is normally the consequence of acute illness, trauma or surgery and leads to reduced renal function [28]. 
Nurses can identify those at risk of AKI:

- Age 65 years or over.

- History of acute kidney injury.

- Chronic kidney disease.

- Symptoms of urological conditions.

- Chronic conditions such as heart failure, liver disease and diabetes mellitus.

- Neurological or cognitive impairment or disability (which may limit independent fluid intake).

- Sepsis.

- Hypovolaemia.

- Oliguria (urine output less than $0.5 \mathrm{~mL} / \mathrm{kg} / \mathrm{h}$ ).

- Nephrotoxic drug use within the last week (especially if hypovolaemic) e.g. nonsteroidal anti-inflammatory drugs (NSAIDs), angiotensin-converting enzyme (ACE) inhibitors, angiotensin II receptor antagonists (ARBs) and diuretics.

- Exposure to iodinated contrast agents within the past week.

- Cancer and cancer therapy.

- Immunocompromise (e.g. HIV infection).

- Toxins (e.g. some herbal remedies, poisonous plants and animals).

Risk should be documented and discussed within the MDT and an enhanced plan of care initiated. In at-risk patients baseline assessment of blood creatinine should be conducted with daily monitoring in the peri-operative period and continued regularly throughout admission. Medication review should be conducted pre-operatively and, in the peri-operative period, identification of nephrotoxic drugs and those that may significantly reduce BP during acute illness.

Management of AKI depends on the cause. Nursing interventions include:

1. Identifying those at risk/presenting with AKI, reporting to senior clinicians.

2. Accurate recording and documentation of fluid balance.

3. Maintaining fluid intake.

4. Acting as a conduit of information between the MDT and the patient and family. In the frailest patients, where the risk of AKI is highest, patient/carer involvement is essential in ensuring adequate fluid intake.

Constipation can be acute or chronic and is a common complication following fracture and during periods of ill health/immobility. Prevention should be considered early in the care pathway. Prevention of constipation from a nursing perspective should involve:

- Regular assessment of bowel function including frequency and consistency of defaecation.

- Providing and encouraging a palatable fibre-rich diet and prevention of dehydration.

- Careful but early use of prescribed aperients. 


\subsubsection{Healthcare Associated Infection}

\subsubsection{Pneumonia: Nursing Assessment, Prevention and Management}

Nursing interventions to prevent pneumonia reflect general good nursing practice for the older people:

- Universal infection prevention precautions.

- Good pain relief to facilitate coughing, deep breathing and mobility.

- Early, regular mobilisation; encouraging activity out of bed.

- Prevention of aspiration risks.

- Encourage patients to sit in a chair for meals.

- Monitoring of dysphagia and swallow and cough reflexes.

- Thickener in drinks or modified diets as appropriate.

- Education of family/carers about the risk and preventive strategies in use.

- Reporting of signs and symptoms of respiratory problems to medical practitioners.

Patients with pneumonia need to be closely monitored by the nursing team for further deterioration. Adequate nutrition is central to supporting recovery and enteral feeding may be needed, although nasogastric feeding can increase the risk of aspiration. Hydration, early mobilisation, encouraging deep breathing and coughing, regular changes of position, chest physiotherapy and nebulisers to moisten secretions can also assist in recovery.

\subsubsection{Urinary Tract Infection: Nursing Assessment, Prevention and Management}

Prevention, recognition and management of urinary tract infection (UTI) are the responsibility of the whole team, but are a fundamental nursing role. Strategies for prevention, risk reduction and recognition of UTI include:

- Avoiding indwelling urinary catheters as much as possible.

- Insertion and removal of urinary catheters under aseptic conditions.

- Using a closed drainage catheter bag system.

- Compliance with infection prevention precautions when inserting, handling and removing catheters.

- Meticulous perineal hygiene.

- Removal of indwelling urinary catheters as soon as possible.

- Reducing the risk of dehydration.

- Early mobilisation to reduce urinary stasis.

- Monitoring for signs of developing infection; particularly delirium, fever and tachycardia.

- Obtain a urine sample for microbiological analysis and referral to medical staff if symptoms of urinary tract infection are present. 


\subsubsection{Venous Thromboembolism: Nursing Interventions for Prevention}

Following hip fracture there is a high risk of venous thromboembolism (VTE). Although prevention and medical management of VTE are considered in Chap. 7, the nursing role in prevention includes assessment of risk on admission and when the patient's condition changes using a risk assessment tool. Nursing specific measures that contribute to the prevention of VTE include:

- Restoration of mobility.

- Supporting early mobilisation and leg exercises to activate the calf muscle pump.

- Maintaining adequate hydration.

- Patient and carer information about the causes, prevention and the need to comply with prophylaxis, especially on discharge/transfer.

- Observation of patients for signs and symptoms of deep vein thrombosis and pulmonary embolism.

Nursing interventions for prophylaxis focus on mechanical measures, particularly graduated compression "anti-embolic" stockings. Stockings can, however, contribute to lower limb compartment syndrome, skin ulceration and common peroneal nerve palsy and should not be used in patients with cardiac or vascular disease, fragile skin or limb shape/deformity preventing correct fit. Safe use of compression stockings includes making sure that stockings are correctly fitted, checking to make sure the fit is not affected by changes in leg shape due to oedema and ensuring stocking are removed regularly for hygiene purposes, assessment of neurovascular status of the limb and checking for skin problems [29].

\subsection{Rehabilitation, Discharge and Continuing Care}

Independence in physical function and quality of life drive multidisciplinary rehabilitation. Effective rehabilitation is important as it promotes independence and supports patients in reaching their potential. Rehabilitation in the hospital setting and beyond tend to be largely a nursing role, alongside therapists, because of the need to support rehabilitation as part of $24 \mathrm{~h}$ and extended care in a collaborative, supportive culture with patient motivation in mind. There is limited evidence, however, regarding the value of different care strategies in supporting rehabilitation and discharge and which team members can best provide such care.

Early supported multidisciplinary rehabilitation can reduce hospital stay, improve early return to function and impact positively on both readmission rates and the level of public-funded home care required [30]. Community rehabilitation schemes facilitate early discharge of less frail patients to their own home. Ongoing rehabilitation allows them to continue to improve functionally and progress towards their goals after discharge, although in many settings this is not provided. 
From admission, patients should be offered a formal, acute, orthogeriatric or orthopaedic ward-based hip fracture programme that includes the following [31]:

- Comprehensive multidisciplinary geriatric/orthogeriatric assessment and continuous review.

- Rapid preoperative optimisation of fitness for surgery.

- Early identification of individual goals for rehabilitation; to recover mobility and independence and facilitate return to pre-fracture residence and long-term well-being.

- Liaison or integration with related services, particularly mental health, falls prevention, bone health, primary care and social services.

- Clinical and service governance responsibility for all stages of the pathway of care and rehabilitation, including those aspects delivered in the community.

Patients with hip fracture have a lower perception of readiness for hospital discharge than other medical-surgical groups [32]. Improvements are needed in nursing practice so that patients feel adequately prepared for discharge and achieve the best possible outcomes. Discharge planning in acute hospitals is multidisciplinary, but nurses take responsibility for much of this, particularly patient and family education, coordination and liaising with follow-on care providers.

The responsibility for care after discharge is often delegated to the patient and their family along with the general practitioner and, sometimes, community care staff. The patient and their caregivers must be able to understand the discharge instructions so that they can recall aftercare instructions and remember to follow them. Providing patients with written discharge instructions has proven to be valuable, as has the provision of information in electronic formats via a smart phone or tablet applications and other devices [33]. Supporting oral information/education with written/electronic information on discharge helps older people with perception, visual, auditory and/or cognitive problems to manage the complex multiple messages.

In an ideal world, patients should have the option to receive rehabilitative care in a specialist rehabilitation setting. This is not an option in most resource-poor settings and is rarely the case even in settings with better resources. The fundamental goal of orthogeriatric care is to discharge the patient to either independent or supported living in their own home or to alternative accommodation where postdischarge care can be provided either permanently or temporarily. Patients and families need intensive support when returning to a community setting or moving to residential care following discharge. Issues that need to be considered include the prevention of future falls, the continued management of bone fragility and secondary fracture prevention as well as the need for continued progress towards optimum achievement of rehabilitation. Post-discharge services vary significantly globally, and the availability of specialist community nursing resources is even more of a challenge than in the hospital setting.

Nurses must be aware of the possibility and effects of care giver burden especially since the majority of orthogeriatric patients require physical care after 
discharge. Care giver burden is defined as "the physical, emotional and financial responses of a caregiver to the changes and demands caused by providing help to another person with a physical or mental disability" [34]. Continued support of care givers should be in place following hospital discharge. Ongoing communication and contact are vital in ensuring care giver burden is minimised. The ability of informal care to meet the care needs of patients is limited, so longer term plans may need to be in place if care needs are prolonged or carers are not able to cope [35].

\subsection{Palliative and End of Life Care}

Hip fractures can herald decline in function and independence and may indicate the beginning of the end of life. Such decline leads to a variety of experiences and symptoms that increase patient need for carefully planned sensitive nursing care. Palliative care is defined by the World Health Organization [36] as: "an approach that improves the quality of life of patients and their families facing the problems associated with life-threatening illness, through the prevention and relief of suffering by means of early identification and impeccable assessment and treatment of pain and other problems, physical, psychosocial and spiritual". This approach should be available for people who are struggling to recover from a hip fracture whether they are likely to be at the end of their lives or require intensive support for management of symptoms and experiences when outcomes from the acute care episode are poor.

The fundamental aims of palliative care include [36]:

- Providing adequate pain relief and minimising discomfort by providing symptom relief.

- Affirming life and regarding dying as a normal process.

- Intending neither to hasten nor postpone death.

- Integrating the psychological and spiritual aspects of patient care.

- Offering a support system to help patients live as actively as possible until death.

- Offering to provide a system of support to help the family cope during the patient's illness and death and in their own bereavement.

- Working collaboratively as a team to address the needs of patients and their families, including bereavement counselling, if indicated.

- Enhancing quality of life and positively influencing the course of illness.

- Applicable early in the course of illness, in conjunction with other therapies that are intended to prolong life, such as chemotherapy or radiation therapy, and includes those investigations needed to better understand and manage distressing clinical complications.

This philosophy allows for physical, psychological, social and emotional care for patients and their families when the patient does not have the physical resilience to fully recover from/survive the trauma of the injury. Typically, palliative 
care is provided by an MDT who focuses on the assessment and treatment of pain and other symptoms whilst ensuring that care is enhanced by patient-centred communication and decision-making across the continuum of care settings, from hospital to home.

Identifying patients for whom a palliative care approach is most appropriate is difficult and is influenced by cultural issues. Nurses play a key role in helping the patient throughout this process. Following hip fracture, patients may have multiple co-morbidities that can additionally limit life, but most recover well from surgery and have good functional outcomes and subsequent quality of life. Appropriate models of end of life care are currently a matter of considerable discussion and debate. Palliative care has not previously been a natural consideration in orthopaedic care, so this is a matter for continuing debate and discussion rather than something that is currently integrated into practice [13].

The patient and family must be included in making decisions that ensure ongoing care and treatment is appropriate to the patient's needs. Good nursing care for those at the end of their life should include physical, emotional and psychological aspects of care along with spiritual support [37]. The process of dying creates multiple emotions and feelings for all involved: the patient, family, carers and the care providers [36]. It is the responsibility of the MDT, through good communication with patients and families, to identify those who were frail pre-fracture and for whom the fall, fracture, surgery and hospitalisation experience may lead to the "winding down" of the body at the end of life.

\subsection{Secondary Prevention, Health Improvement and Health Promotion}

The nursing team are in an ideal position to recognise and act on the need for bone health assessment and identification of those whose fracture requires investigation for osteoporosis (see Chap. 14) as they have the most sustained contact with patients. This, however, is not a simple process since most patients will be unaware of the influence of bone health on their current fracture. Early in the care trajectory it may be a shock to them that the cause of the fracture may be fragile bone due to osteoporosis. While there is, therefore, an opportunity for nurses in any care setting to instigate bone health assessment referral, this needs to be discussed in a sensitive manner with patients and carers. Nurses need to be able to discuss the reasons for referral for bone health assessment with patients as soon as possible after the fracture has occurred so that patients and their families can be prepared for the next steps. Conversations that introduce the topic of bone health and osteoporosis can be conducted during normal nursing care activities in a manner which enables patients to gradually acknowledge the need for them to consider their bone health. To do this, nurses working in acute settings need to have enough understanding of the processes involved in bone health assessment and the treatment of osteoporosis so that they can effectively educate and counsel patients and their families. 


\subsection{Nursing Resources, Education and Leadership}

There is a global shortage of nurses owing to changing demographics, political ideologies and financial austerity and recruitment and retention problems. The availability of appropriately educated and skilled nurses to provide both fundamental and specialist care is a major concern. A large international study [38] demonstrated that an increase in a nurses' workload by one patient, from eight to nine patients per qualified nurse, increased the likelihood of an inpatient dying within 30 days of admission by $7 \%$. Under-resourcing nursing teams leads to insufficient capacity for the team to undertake actions to prevent morbidity and mortality. Studies have indicated that this "missed care" is associated with one or more adverse patient outcomes including; medication errors, urinary-tract infections, falls, pressure injuries, critical incidents, quality of care and patient readmissions. The quality of studies relating to missed care is, however, weak and the link between missed care and mortality is yet to be explored in more robust research [19]. Most of the research has, however, considered high resource settings, while in lower resource settings the impact of limited resources on patients and communities has yet to be explored. In many settings the number of qualified nurses is severely limited and skills development focuses on nursing assistants and other team members.

Unless the nursing resource is protected and grows, the added nursing value needed to continue to improve outcomes and the quality of care for patients with fragility fractures globally will not happen. Sahota and Currie [39] pointed out that: “...looking after hip fracture patients well is a lot cheaper than looking after them badly", the nursing resource is significant in this, meaning that it is important for the multidisciplinary community, through organisations such as the Fragility Fracture Network (FFN), to lobby governments and health and social care providers of to ensure that nurses are able to do undertake their role effectively, aligning the profession to the fourth pillar in the FFN call to action [1].

Nurses work in a professional culture of constant skills and knowledge development through learning and reflection. Many nurses working in orthogeriatric settings, however, are more likely to have been educated solely in the care of adults with musculoskeletal problems rather than to meet the complex needs of older people. Multiple "orthogeriatric" specialist skills that combine orthopaedic care expertise with the care of older people are needed that are based on an in-depth knowledge of the theory underpinning care. Few nurses working with patients with fragility fractures have undertaken education beyond that of their initial nursing qualification and they rely on knowledge and skills developed through generic, rather than specialist, education. This education and skills gap can result in care which does not meet all needs. Specialist orthogeriatric nursing education could have a positive impact on patient outcomes [19]. Many nurses working in orthogeriatric settings are better prepared educationally for the care of adults with musculoskeletal problems than to meet the complex needs of older people. Multiple specialist "orthogeriatric" nursing skills are needed as well as fundamental adult nursing skills. Specialist nursing qualifications in orthogeriatric care do not currently exist, so nurses are obliged to be reflective self-led learners who are able to extend their own knowledge 
of both caring for patients following trauma and the complex care of older people together through reflection.

Multidisciplinary orthogeriatric care can be fragmented and less effective if it is not managed or coordinated effectively; nurses' 24-h presence and detailed knowledge of patient pathways make them ideal care co-ordinators. The complex nursing care requirements of orthogeriatric patients mean that they should have their care led by those who are experts in the field with an intuitive understanding of need. In some settings care and its coordination are led by a specialist nurse or coordinator such as a hip fracture nurse specialist, elderly/elder care nurse specialist, trauma nurse coordinator, nurse practitioner or advanced nurse practitioner. In some countries, multidisciplinary collaborative working has supported the development of advanced nursing roles often operationalised as clinical nurse specialists, nurse practitioners or physicians' assistants who have a variety of skills that are complementary to the MDT and enhance patient care. Care should be supervised and managed by those who have enhanced experience, skills and knowledge in working with older people following fracture so that they perceive care needs holistically and from individual perspectives.

\section{References}

1. Fragility Fracture Network (2018) Global call to action on fragility fractures. https://www. fragilityfracturenetwork.org/cta/

2. International Council of Nurses (ICN) (2010) Definition of nursing. Geneva: ICN. http://www. icn.ch/who-we-are/icn-definition-of-nursing/. Accessed 6 May 2016

3. Ziden L, Scherman H, Wenestam MH, C-G. (2010) The break remains-elderly people's experiences of a hip fracture 1 year after discharge. Disabil Rehabil 32(2):103-113

4. Kondo A, Sada K, Ito Y, Yamaguchi C et al (2014) Difficulties in life after hip fracture and expected hospital supports for patients and families. Int $\mathrm{J}$ OrthopTrauma Nurs 18(4): 191-204

5. MacDonald V, Butler Maher A, Mainz H et al (2018) Developing and testing an international audit of quality indicators for older adults with fragility hip fracture. Orthop Nurs 37(2):115-121

6. Pitzul K, Munce S, Perrier L et al (2017) Scoping review of potential quality indicators for hip fracture patient care. BMJ Open 7:e014769. https://doi.org/10.1136/bmjopen-2016-014769

7. Heslop L, Liu S (2014) Nursing-sensitive indicators: a concept analysis. J Adv Nurs 70(11):2469-2482. http://www.ncbi.nlm.nih.gov/pmc/articles/PMC4232854/. Accessed 6 May 2016

8. Meehan AJ, Hommel A, Hertz K et al (2016) Care of the older adult with fragility hip fracture. In: Boltz M, Capezuti E, Fulmer T, Zwicker D (eds) Evidence-based geriatric nursing protocols for best practice. Springer, New York

9. Meehan A, Maher A, Brent L et al (2019) The International Collaboration of Orthopaedic Nursing (ICON): best practice nursing care standards for older adults with fragility hip fracture. Int J Orthop Trauma Nurs 32:3-26

10. Geszar B et al (2017) Hip fracture; an interruption that has consequences four months later. A qualitative study. Int J Orthop Trauma Nurs 26:43-48 
11. Jensen CM, Hertz K, Mauthner O (2018) Orthogeriatric nursing in the emergency and perioperative in-patient setting. In: Hertz K, Santy-Tomlinson J (eds) Fragility fracture nursing. Springer, Cham, pp 53-65

12. Weissenberger-Leduc M, Zmaritz M (2013) Nursing care for the elderly with hip fracture in an acute care hospital. Wien Med Wochenschr 163(19-20):468-475

13. Hertz K, Santy-Tomlinson J (2014) Fractures in the older person. In: Clarke S, Santy-Tomlinson $\mathrm{J}$ (eds) Orthopaedic and trauma nursing: an evidence-based approach to musculoskeletal care. Wiley Blackwell, Oxford, pp 236-250

14. Manning E, Gagnon M (2017) The complex patient: a concept definition. Nurs Health Sci 19:13-21

15. Rankin J, Regan S (2004) Complex needs: the future of social care. Institute for Public Policy Research/Turning Point, London. http://www.ippr.org/files/images/media/files/publication/2011/05/Meeting_Complex_Needs_full_1301.pdf?noredirect=1. Accessed 6 May 2016

16. Hertz K, Santy-Tomlinson J (2017) The nursing role. In: Falaschi P, Marsh D (eds) Orthogeriatrics. Springer, Cham, pp 131-144

17. Marques A, Queiros C (2018) Frailty, sarcopenia and falls. In: Hertz K, Santy-Tomlinson J (eds) Fragility fracture nursing. Springer, Cham, pp 15-26

18. Morley J, Vellas B, van Kan G, Anker S et al (2013) Frailty consensus: a call to action. J Am Med Dir Assoc 14(6):392-397

19. Brent L, Hommel A, Maher AB, Hertz K, Meehan AJ, Santy-Tomlinson J (2018) Nursing care of fragility fracture patients. Injury 49(8):1409-1412

20. Maxwell C, Wang J (2017) Understanding frailty: a nurse's guide. Nurs Clin N Am 52:349-361

21. Bjorkelund KB, Hommel A, Thorngren KG et al (2011) The influence of perioperative care and treatment on the 4-month outcome in elderly patients with hip fracture. AANA J 79(1):51-61

22. Alzheimer's Society (UK) (2010) This is me. London Alzheimer's Society/Royal College of Nursing. https://www.alzheimers.org.uk/site/scripts/download_info.php?downloadID=399. Accessed 6 May 2016

23. Obideyi A, Srikantharajah I, Grigg L, Randall A (2008) Nurse administered fascia iliaca compartment block for pre-operative pain relief in adult fractured neck of femur. Acute Pain 10(3-4):145-149

24. Cross J (2018) Nursing the patient with altered cognitive function. In: Hertz K, SantyTomlinson J (eds) Fragility fracture nursing. Springer, Cham, pp 109-124

25. NICE (National Institute for Health and Care Excellence) (2014) Delirium (QS63). www.nice. org.uk/guidance/qs63. Accessed 6 May 2016

26. NPIAP-EPUAP-PPPIA (National Pressure Injury Advisory Panel/European Pressure Ulcer Advisory Panel/Pan Pacific Pressure Injury Alliance) (2019) Prevention and Treatment of Pressure Ulcers/Injuries: Quick Reference Guide. 3rd Edition file:///C:/Users/tomli/ Downloads/ggg-quick-reference-guide-version29dec2019-secured.pdf

27. Hommel A, Bjorkelund KB, Thorngren K-G, Ulander K (2007) A study of a pathway to reduce pressure ulcers in patients with a hip fracture. J Orthop Nurs 11(3-4):151-159

28. NICE (National Institute for Health and Care Excellence) (2014) Acute kidney injury: Quality standard www.nice.org.uk/guidance/qs76

29. Wellington B, Flynn S, Duperouzel W (2015) Anti-embolic stockings for the prevention of VTE in orthopaedic patients: a practice update. Int J Orthop Trauma Nurs 19(1):45-49

30. Dyer S et al (2017) Rehabilitation following hip fracture. In: Falaschi P, Marsh D (eds) Orthogeriatrics. Springer, Cham, pp 145-163

31. Barberi S, Mielli L (2018) Rehabilitation and discharge. In: Hertz K, Santy-Tomlinson J (eds) Fragility fracture nursing. Springer, Cham, pp 125-113

32. Brent L, Coffrey A (2013) Patient's perceptions of their readiness for discharge following hip fracture surgery. Int J Orthop Trauma Nurs 17:190-198

33. Jensen CM, Overgaard S, Wiil UK, Clemensen J (2019) Can tele-health support self-care and empowerment? A qualitative study of hip fracture patients' experiences with testing an "App". SAGE Open Nurs 5:1-11 
34. Pearlin LI, Mullan JT, Semple SJ, Skaff MM (1990) Caregiving and the stress process: an overview of concepts and their measures. Gerontologist 30(5):583-594

35. Falaschi P, Eleuteri S (2017) The psychological health of patients and their caregivers. In: Falaschi P, Marsh D (eds) Orthogeriatrics. Springer, Cham, pp 201-211

36. World Health Organization (2014) Global atlas of palliative care at the end of life. www.who. int/nmh/Global_Atlas_of_Palliative_Care.pdf

37. Brent L, Santy-Tomlinson J, Hertz K (2018) Family partnerships, palliative care and end of life. In: Hertz K, Santy-Tomlinson J (eds) Fragility fracture nursing. Springer, Cham, pp 137-146

38. Aiken L, Sloane D, Bryneel L, Van den Heede K et al (2014) Nurse staffing and education and hospital mortality in nine European countries: a retrospective observational study. Lancet 383(993): 1824-1830

39. Sahota O, Currie C (2008) Hip fracture care: all change. Editorial. Age Ageing 37:128-129

Open Access This book is licensed under the terms of the Creative Commons AttributionNonCommercial-NoDerivatives 4.0 International License (http://creativecommons.org/licenses/ by-nc-nd/4.0/), which permits any noncommercial use, sharing, distribution and reproduction in any medium or format, as long as you give appropriate credit to the original author(s) and the source, provide a link to the Creative Commons license and indicate if you modified the licensed material. You do not have permission under this license to share adapted material derived from this book or parts of it.

The images or other third party material in this book are included in the book's Creative Commons license, unless indicated otherwise in a credit line to the material. If material is not included in the book's Creative Commons license and your intended use is not permitted by statutory regulation or exceeds the permitted use, you will need to obtain permission directly from the copyright holder.

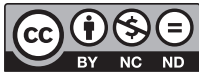

